\title{
Liste abgekürzt zitierter Literatur ${ }^{1}$
}

CCSG $=$ Corpus Christianorum, Series Graeca, Turnhout-Leuven 1977-. $\mathrm{CFHB}=$ Corpus Fontium Historiae Byzantinae, Athen-Berlin-Brüssel-Paris-Rom-Thessaloniki-Washington-Wien 1967-.

CparG $=$ Corpus Paroemiographorum Graecorum. I. Ediderunt E.L. A LEUTSCH et F.G. SCHNEIDEWIN. II. Edidit E.L. A LEUTSCH, Gottingae 1839. 1851.

CPG = Clavis Patrum Graecorum cura et studio M. GEERARD, I-V, Turnhout 1983.1974.1979.1980.1987; Clavis Patrum Graecorum. Supplementum cura et studio M. GEERARD et J. NORET, adiuvantibus F. GLORIE et J. DESMET, Turnhout 1998; Clavis Patrum Graecorum, Volumen III. Editio secunda, anastatica, addendis locupletata a J. NORET, Turnhout 2003; Clavis Patrum Graecorum, Volumen IV. Concilia. Catenae. Cura et studio M. GEERARD. Deuxième édition, revue et mise à jour par J. NORET, Turnhout 2018.

$\mathrm{CPL}=$ Clavis Patrum Latinorum, qua in Corpus Christianorum edendum optimas quasque scriptorum recensiones a Tertulliano ad Bedam commode recludit E. DEKKERS, opera usus qua rem praeparavit et iuvit Aem. GAAR $\dagger$. Editio tertia aucta et emendata, Steenbrugis 1995.

$\mathrm{CSCO}=$ Corpus Scriptorum Christianorum Orientalium, Parisiis-Lovanii 1903-.

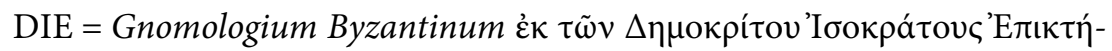
tov e variis codicum exemplis restitutum, in: Studien zu den griechischen Florilegien von C. WACHSMUTH, Berlin 1882, 166-270.

GCS $=$ Die griechischen christlichen Schriftsteller der ersten (drei) Jahrhunderte, Leipzig-Berlin 1897-.

GNO = Gregorii Nysseni Opera , auxilio aliorum virorum doctorum edenda curavit W. JAEGER..., Leiden 1952-.

LXX = Septuaginta. Vetus Testamentum Graecum Auctoritate Academiae Scientiarum Gottingensis editum, Göttingen 1952-.

NT $=$ Novum Testamentum graece. Begründet von Eberhard und Erwin NestLE, herausgegeben von B. und K. Aland, J. KaRAvidopoulos, C.M. MARTINI, B.M. METZGER. Herausgegeben vom Institut für Neutestamentliche Textforschung, Stuttgart ${ }^{28} 2012$.

\footnotetext{
${ }^{1}$ Für die bibliographischen Angaben der Editionen, die für die Identifikation der Zitate herangezogen wurden, sei auf den Index locorum in diesem Band verwiesen.
} 
PG = Patrologiae cursus completus... Series Graeca..., accurante et recognoscente J.-P. Migne, 161 vol., Parisiis 1857-1866.

PO = Patrologia Orientalis, Paris 1903-.

PTS = Patristische Texte und Studien, Berlin 1964-.

RAC $=$ Reallexikon für Antike und Christentum, Stuttgart 1950-.

SChr $=$ Sources Chrétiennes, Paris 1942-.

SJD $=$ Die Schriften des Johannes von Damaskos (Patristische Texte und Studien), Berlin 1969-.

Antiquitatum Iudaicarum epitoma = Flavii Iosephi Antiquitatum Iudaicarum epitoma edidit B. NIESE, Marburgi Chattorum 1887-1896.

AUZÉPY, Étienne et Jean = M.-Fr. AUZÉPY, De la Palestine à Constantinople (VIII ${ }^{e}-I X^{e}$ siècles): Étienne le Sabaïte et Jean Damascène, in: Travaux et Mémoires, 12, Paris 1994, 183-218.

AUZÉPY, Histoire = M.-Fr. AUZÉPY L'histoire des iconoclastes (Bilans de recherche, 2), Paris 2007 (darin Wiederabdruck des vorigen)

BARDENHEWER, Geschichte $=$ O. BARDENHEWER, Geschichte der altkirchlichen Literatur, I-V, Freiburg im Breisgau ${ }^{2} 1902 .{ }^{2} 1914 .{ }^{2} 1923 .{ }^{2} 1924.1932$.

Billius = Sancti Ioannis Damasceni opera, multo quam unquam antehac auctiora, magnaque ex parte nunc de integro conuersa. Per I. BILliUM..., Parisiis 1577.

BRENT, Hippolytus = A. BRENT, Hippolytus and the Roman Church in the Third Century. Communities in Tension Before the Emergence of a Monarch-Bishop (Supplements to Vigiliae Christianae, XXXI), Leiden-New York-Köln 1995.

CANART, Codices 1745-1962 = P. CANART, Codices Vaticani Graeci. Codices 1745-1962. Tomus I. Codicum enarrationes (Bibliothecae apostolicae Vaticanae codices manu scripti recensiti), In bibliotheca Vaticana 1970.

CANART, Études $=$ P. CANART, Études de paléographie et de codicologie. Reproduites avec la collaboration de M.L. AgATI et M. D'Agostino (Studi e testi, 450-451), Città del Vaticano 2008.

CASTELLI, Ricerche $=$ E. CASTELLI, Un falso letterario sotto il nome di Flavio Giuseppe. Ricerche sulla tradizione del Пepi tov ravtó e sulla produzione letteraria cristiana a Roma nei primi decenni del III secolo (Jahrbuch für Antike und Christentum. Ergänzungsband. Kleine Reihe, 7), Münster/ Westfalen 2011.

Chryssavgis, John Climacus = J. Chryssavgis, John Climacus: From the Egyptian desert to the Sinaite mountain, Aldershot-Burlington 2004. 
DALEY, Leontius $=$ Leontius of Byzantium. Complete Works. Edited and Translated, with an Introduction, by Br.E. DALEY, Oxford 2017.

Declerck = Die Schriften des Johannes von Damaskos, VIII / 6. Iohannis monachi (VII saeculo ineunte) Sacra (olim Iohanni Damasceno attributa). Liber II. De rerum humanarum natura et statu. Zweite Rezension. Erster Halbband. A-E ( $\left.{ }^{\star} \mathrm{II}^{2} 1-1592\right)$. Besorgt von J. DECLERCK (Patristische Texte und Studien, 76), Berlin-Boston 2018.

DECLERCK, À qui la lettre = J. DECLERCK, À qui la lettre Sur les décrets de Nicée d'Athanase d'Alexandrie (CPG 2120) était-elle adressée? in: Philologie, herméneutique et histoire des textes entre Orient et Occident. Mélanges en hommage à Sever J. Voicu édités par Fr.P. BARONE, C. MACÉ, P.A. UBIERNA (Instrumenta patristica et mediaevalia, 73), Turnhout 2017, 381-404. DeCleRCK, Retour $=$ J. DeCleRCK, Les Sacra Parallela nettement antérieurs à Jean Damascène: retour à la datation de Michel Le Quien. Byzantion 85 (2015), 27-65.

DeCleRCK, Un manuscrit pour Basile ${ }^{e r}=\mathrm{J}$. DeCleRCK, Le Parisinus gr. 923: un manuscrit destiné à l'empereur Basile I ${ }^{e r}$ (867-886). Byzantion 87 (2017), 181-206.

DEVREESSE, Le fonds Coislin = Bibliothèque nationale. Département des manuscrits. Catalogue des manuscrits grecs. II. Le fonds Coislin par R. DEVREESSE, Paris 1945.

DIEKAMP, Doctrina = Doctrina Patrum de Incarnatione Verbi. Ein griechisches Florilegium aus der Wende des siebenten und achten Jahrhunderts zum ersten Male vollständig herausgegeben und untersucht von F. DIEKAMP, Münster in Westf. 1907. 2. Auflage mit Korrekturen und Nachträgen von B. PhanOURGaKis, herausgegegeben von E. ChrYsos, Münster 1981.

Eastern Christianity: Greek = Languages and Cultures of Eastern Christianity: Greek. Edited by S.F. JoHNSON (The Worlds of Eastern Christianity, 300-1500, Vol. 6), Farnham-Burlington, 2015.

GRIBOMONT, Nil = J. GRIBOMONT, La tradition manuscrite de saint Nil. I: La correspondance. Studia monastica XI (1969), 231-267.

Haidacher $=\mathrm{S}$. HAIDACHER, Chrysostomos-Fragmente im Maximos-Florilegium und in den Sacra Parallela. Byzantinische Zeitschrift 16 (1907), 168201.

HARRIS, Fragments $/$ Harris = Fragments of Philo Judœus Newly Edited by J. RENDEL HARRIS, Cambridge 1886. 
HoLl, Fragmente $/$ Holl, n $1-503$ = Fragmente Vornicänischer Kirchenväter aus den Sacra parallela herausgegeben von K. Holl (Texte und Untersuchungen, N. F. V. Band, Heft 2), Leipzig 1899.

Holl, Sacra Parallela = Die Sacra Parallela des Johannes Damascenus von K. Holl (Texte und Untersuchungen, N. F. Erster Band, Heft 1), Leipzig 1896.

IHM, Ps.-Maximus Confessor = S. IHM, Ps.-Maximus Confessor. Erste kritische Edition einer Redaktion des sacro-profanen Florilegiums Loci communes nebst einer vollständigen Kollation einer zweiten Redaktion und weiterem Material (Palingenesia, LXXIII), Stuttgart 2001.

KAEGI, Heraclius = W.E. KAEGI, Heraclius: Emperor of Byzantium, Cambridge 2003.

KontoumA, John of Damascus = V. KonTOumA, John of Damascus. New studies on his Life and Works, Farnham-Burlington 2015.

Lequien = Sancti Patris Nostri Joannis Damasceni, monachi et presbyteri Hierosolymitani opera omnia qua exstant, et ejus nomine circumferuntur. Ex variis editionibus, et codicibus manu exaratis, Gallicanis, Italicis \& Anglicis, collecta, recensita, Latine versa, atque annotationibus illustrata, cum proviis Dissertationibus, \& copiosis indicibus. Opera et studio M. LEQUIEN, t. II, Parisiis 1712 et Venetiis (Editio novissima Veneta longe aliis accuratior) 1748, 278-790.

Lewy $=$ Neue Philontexte in der Überarbeitung des Ambrosius. Mit einem Anhang: Neu gefundene griechische Philonfragmente. Von H. LEWY (Sitzungsberichte der Preußischen Akademie der Wissenschaften. Phil.-hist. Klasse. 1932. IV), Berlin 1932, 23-84.

LOOFS, Studien = Studien über die dem Johannes von Damaskus zugeschriebenen Parallelen von Fr. LOOFs, Halle 1892.

LOUTH, John Damascene = A. LOUTH, St John Damascene. Tradition and Originality in Byzantine Theology, Oxford 2002.

MAKSIMCZUK, Florilegium Hierosolymitanum = J. MAKSIMCZUK, The Textual Tradition of the Florilegium Hierosolymitanum (and its Relations with the Florilegium Coislinianum). Jahrbuch der Österreichischen Byzantinistik 67 (2017), 81-101.

MALLEY, Four Unedited Fragments = W.J. MALLEY, Four Unedited Fragments of the De universo of the Pseudo-Josephus Found in the Chronicon of George Hamartolus (Coislin 305). Journal of Theological Studies, N.S. XVI (1965), 13-25.

Mangey $=$ Philonis Judoi Opera quoe Reperiri potuerunt Omnia. Textum cum MSS. contulit... Th. MANGEY, t. II, Londinii 1742, 648-674. 
Ps.-Max. Conf., Loci communes = S. IHM, Ps.-Maximus Confessor. Erste kritische Edition einer Redaktion des sacro-profanen Florilegiums Loci communes nebst einer vollständigen Kollation einer zweiten Redaktion und weiterem Material (Palingenesia, LXXIII), Stuttgart 2001.

MunitiZ, Accounts = J.A. MUNITIZ, Synoptic Accounts of the Seventh Council. Revue des études byzantines 32 (1974), 147-186.

NAUTIN, Hippolyte = P. NAUTIN, Hippolyte. Contre les hérésies. Fragment. Étude et édition critique (Études et textes pour l'histoire du dogme de la Trinité, 2), Paris 1949.

NAUTIN, Josipe = P. NAUTIN, Hippolyte et Josipe. Contribution à l'histoire de la littérature chrétienne du troisième siècle (Études et textes pour l'histoire du dogme de la Trinité, 1), Paris 1947.

NODET, Sommaires $=\mathrm{E}$. NODET, Texte et sommaires des Antiquités de Josèphe. Éditions multiples (Cahiers de la Revue biblique, 89), Leuven-ParisBristol/CT, 2017.

Nodet, Texte = E. NodeT, Le texte des Antiquités de Josèphe (l.1-10). Revue biblique 94 (1987), 323-375.

RICHARD, Florilèges spirituels $=\mathrm{M}$. RICHARD, Florilèges <spirituels $>$ grecs (Dictionnaire de Spiritualité ascétique et mystique, fasc. 33-34, Paris 1962, Sp. 476-486 [= Opera minora, t. I, Turnhout-Leuven 1976, n 1]).

Royse $=$ J.R. RoYSE, Reverse Indexes to Philonic Texts in the Printed Florilegia and Collections of Fragments (Brown Judaic Studies, 287. The Studia Philonica Annual. Studies in Hellenistic Judaism, V), Atlanta/Georgia 1993. Royse, Spurious Texts = The Spurious Texts of Philo of Alexandria. A Study of Textual Transmission and Corruption with Indexes to the Major Collections of Greek Fragments by J.R. ROYSE (Arbeiten zur Literatur und Geschichte des Hellenistischen Judentums, XXII), Leiden-New York-København-Köln 1991.

SCHADLER, John of Damascus = P. SCHADLER, John of Damascus and Islam. Christian Heresiology and the Intellectual Background to Earliest ChristianMuslim Relations (History of Christian-Muslim Relations, 34), Leiden-Boston 2018.

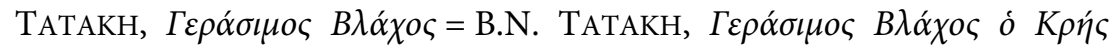

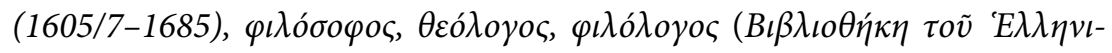

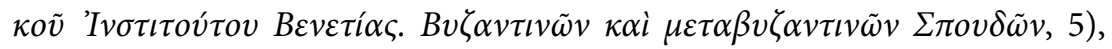
Bevetía 1973.

Theophan. Conf. $=$ Theophanis chronographia. Recensuit C. DE BOOR. Volumen I textum graecum continens, Lipsiae 1888. 
The Sabaite Heritage = The Sabaite Heritage in the Orthodox Church from the Fifth Century to the Present edited by J. PATRICH (Orientalia Lovaniensia Analecta, 98), Leuven 2001.

Thum = Die Schriften des Johannes von Damaskos, VIII / 4. Iohannis monachi (VII saeculo ineunte) Sacra (olim Iohanni Damasceno attributa). Liber II. De rerum humanarum natura et statu. Erste Rezension. Erster Halbband. A-E (II $1-1000)$. Besorgt von T. THUM (Patristische Texte und Studien, 74), Berlin-Boston 2018.

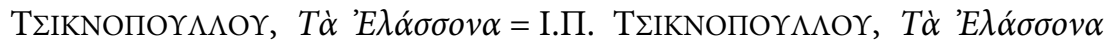

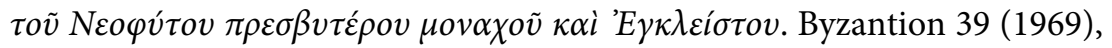
318-419.

Vita Michaelis Syncelli = The Life of Michael the Synkellos. Text, Translation and Commentary by M.B. CunNingHAM (Belfast Byzantine Texts and Translations, 1), Belfast 1991.

Vita Stephani iunioris = La Vie d'Étienne le Jeune par Étienne le Diacre. Introduction, édition et traduction M.-Fr. AUZÉPY (Birmingham Byzantine and Ottoman Monographs, 3), Birmingham 1997.

WAHL, Deuteronomium-Text $=$ Der Deuteronomium-Text der Sacra Parallela. Von O. WAHL (Nachrichten der Akademie der Wissenschaften in Göttingen. I. Philologisch-Historische Klasse. Jahrgang 1997. Mitteilungen des Septuaginta-Unternehmens, XXIII), Göttingen 1997, 111-156.

WAHL, 3 Könige-Text = O. WAHL, Die Sacra-Parallela-Zitate aus den Büchern Josua, Richter, 1/2 Samuel, 3/4 Könige sowie 1/2 Chronik (Abhandlungen der Akademie der Wissenschaften zu Göttingen. Philologisch-Historische Klasse. Dritte Folge, Band 255. Mitteilungen des Septuaginta-Unternehmens, XXIX), Göttingen 2004.

WAHL, 4 Könige-Text vide sub WAHL, 3 Könige-Text

WAHL, Prophetenzitate = O. WAHL, Die Prophetenzitate der Sacra Parallela in ihrem Verhältnis zur Septuaginta-Textüberlieferung (Studien zum Alten und Neuen Testament, XIII), München 1965.

WAHL, Proverbien-Text $=$ O. WAHL, Der Proverbien- und Kohelet-Text der Sacra Parallela (Forschung zur Bibel, 51), Würzburg 1985.

WAHL, Richter-Text vide sub WAHL, 3 Könige-Text

WAHL, 1 Samuel-Text vide sub WAHL, 3 Könige-Text

WAHL, 2 Samuel-Text vide sub WAHL, 3 Könige-Text

WAHL, Sirach-Text $=$ O. WAHL, Der Sirach-Text der Sacra Parallela (Forschung zur Bibel, 16), Würzburg 1974.

WAHL, 1 Chronik-Text vide sub WAHL, 3 Könige-Text 
Weitzmann, Miniatures $=$ The Miniatures of the Sacra Parallela. Parisinus graecus 923 by K. WeItZMANN (Studies in Manuscript Illumination, 8), Princeton/New Jersey 1979. 
